\title{
Post-tuberculosis mortality and morbidity: Valuing the hidden epidemic
}

Matthew Quaife, Faculty of Public Health and Policy, and TB Modelling Group, TB Centre, London School of Hygiene and Tropical Medicine, London, UK

Rein MGJ Houben, TB Modelling Group, TB Centre, London School of Hygiene and Tropical Medicine, London, UK

Brian Allwood, Division of Pulmonology, Department of Medicine, Stellenbosch University, South Africa

Ted Cohen, Department of Epidemiology of Microbial Diseases, Yale School of Public Health, New Haven, USA

Anna K. Coussens, Infectious Diseases and Immune Defence Division, Walter and Eliza Hall Institute of Medical Research, Parkville, Australia

Anthony D. Harries, Centre for Operational Research, International Union Against Tuberculosis and Lung Disease, Paris, France

Sanne van Kampen, National eHealth Living Lab, Department of Public Health and Primary Care, Leiden University Medical Center, the Netherlands

Florian Marx, DST-NRF Centre of Excellence in Epidemiological Modelling and Analysis (SACEMA), Faculty of Science \& Desmond Tutu TB Centre, Department of Paediatrics and Child Health, Faculty of Medicine and Health Sciences, Stellenbosch University, Cape Town, South Africa

Sedona Sweeney, Faculty of Public Health and Policy, London School of Hygiene and Tropical Medicine, London, UK

Robert S. Wallis, Aurum Institute, Johannesburg

Nicolas A Menzies, Department of Global Health and Population, Harvard T H Chan School of Public Health, Boston, MA, USA 
Case fatality rates for tuberculosis disease (TB) have fallen progressively over the past 20 years, and an estimated 54 million people have survived TB since $2000 .{ }^{1}$ More recently there have been increasing efforts to understand the long-term implications of morbidity and mortality posttuberculosis, and a growing body of evidence describes how successful completion of treatment is unlikely to represent the end of ill health. ${ }^{2}$

A recent meta-analysis estimated all-cause mortality to be 2.91 (95\% Cl: $2.21-3.84)$ times greater among individuals post-tuberculosis compared to age- and sex-matched controls. ${ }^{3}$ While these estimates may be inflated by coalescing factors predisposing to both TB and early mortality, separate lines of research have described the causal pathways through which TB impacts future health. These long-term post-tuberculosis sequelae include substantial morbidity from residual tissue damage, despite microbiologic cure. Evidence from meta-analysis suggests that pulmonary TB is an independent risk factor for airflow obstruction and spirometric restriction ${ }^{4}$, alongside chronic obstructive pulmonary disease (COPD) $)^{5}$. TB meningitis and musculoskeletal TB also cause substantial long-term morbidity, and individuals post-tuberculosis face elevated risks of recurrent TB. ${ }^{6}$

However, chronic impairments are not yet reflected in conventional measures of TB burden ${ }^{7}$, or in analyses comparing TB policy options. ${ }^{8,9}$ Here we describe how the burden of post-tuberculosis mortality and morbidity can be quantified using the disability-adjusted life year (DALY) framework. We provide an example of how this can change TB burden estimates in a high-incidence setting and consider the consequences for TB interventions, programming, and future research.

DALYs are a composite measure of health loss widely used to evaluate the impact and costeffectiveness of health programmes. Interventions seek to avert DALYs, which are the sum of years of life lost due to premature death and years lived with disability. ${ }^{7}$ In a typical analysis, DALYs averted by TB interventions only account for the mortality and morbidity accruing during treatment of TB disease, assuming that survivors return to full health post-tuberculosis. ${ }^{9}$ Although some conditions have disability weights representing chronic disability following resolution of acute disease (e.g. long term consequences of stroke), TB does not. ${ }^{1}$

As an example, we considered pulmonary TB in India and calculated conservative estimates based only on post-tuberculosis changes in COPD prevalence only (Figure 1). For active TB DALYs, we assumed a 2-year duration of disability and case fatality estimates reported by $\mathrm{WHO} .{ }^{10}$ To calculate post-tuberculosis years of life lost, we estimated a lower-bound mortality rate ratio (MRR) of 1.22 based on elevated COPD prevalence and mortality among post-tuberculosis individuals. ${ }^{5}$ For post-TB morbidity we estimated a post-tuberculosis disability weight of 0.053 based on elevated posttuberculosis COPD prevalence and published disability weights for COPD and other chronic respiratory diseases.

We find that if post-tuberculosis mortality and morbidity is considered, the estimated burden of TB in India due to incident TB in 2018 increases by an additional 6.1 million DALYs, a 54\% increase on estimates that assume a return to full health at the end of TB treatment. This burden would further increase, if post-tuberculosis conditions other than COPD were considered. We estimate an additional $20.1 \mathrm{~m}$ DALYS (a $174 \%$ increase) as upper-bound, assuming that all excess mortality was attributable to past TB (MRR of 2.91) .

One implication of accounting for post-tuberculosis ill-health is a change in the prioritisation of TB programming. For example, the impact and cost-effectiveness of preventative interventions will increase due to greater DALYs averted per TB episode prevented - particularly relevant in the context of recent vaccine trials, and high-level political commitments to provide preventative 
therapy to 30 million individuals by $2022 .{ }^{11}, 12$ Interventions that limit lung damage from TB disease should also receive increased attention, including tools allowing earlier TB diagnosis ${ }^{13}$, and new regimens that protect lung function during TB treatment. ${ }^{14}$

Given this potentially large burden of disease associated with post-tuberculosis, several lines of future research are important. First, clinical evidence on post-tuberculosis points to a highly heterogeneous set of symptoms and conditions, which need to be understood to enable more tailored post-tuberculosis care. Second, there is little evidence on how mortality and morbidity exacerbate household and macro-economic consequences of TB, since current estimates assume no costs are incurred and no productivity is diminished after treatment. Third, consistent measurement of lung damage could be used to quantify whether case finding is diagnosing cases earlier in their disease, which in turn relates to preventing transmission. ${ }^{15}$ Fourth, estimates of mortality and morbidity from meta-analyses remain subject to bias due to confounding, and work is needed to confirm the causal impact of post-tuberculosis on long-term morbidity and mortality.

While much remains unknown, post-tuberculosis morbidity and mortality are a significant part of TB natural history, which deserve greater integration into the END TB strategy. As TB policies focus on both care and prevention, research and policy makers cannot ignore the impact and needs of individuals after resolution of TB disease. ${ }^{12}$

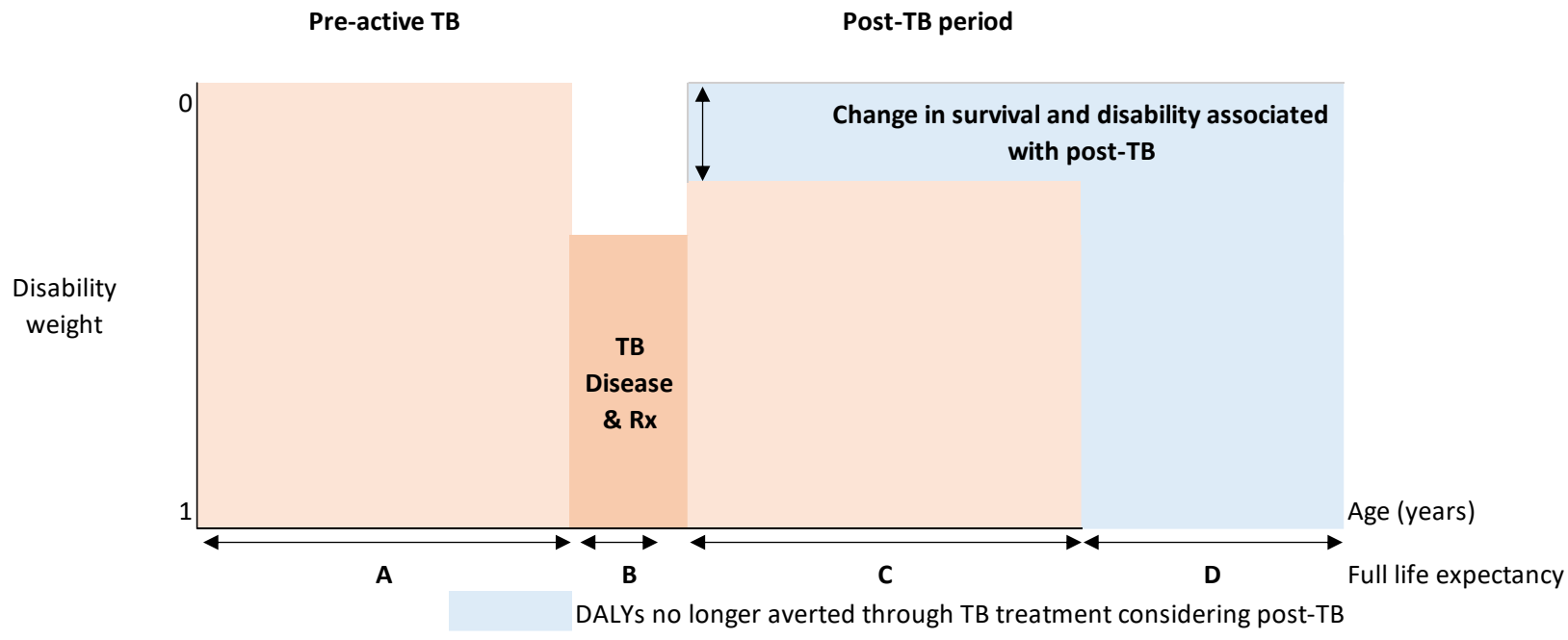

Figure 1: Demonstration of post-tuberculosis DALY loss for a typical survivor of pulmonary tuberculosis treatment in India without HIV infection. Shaded blue area does not consider mortality or morbidity from conditions which share common causes with tuberculosis, thus this figure refers to a lower-bound scenario of post-tuberculosis. 


\section{Reference list}

1. Kyu HH, Maddison ER, Henry NJ, et al. Global, regional, and national burden of tuberculosis, 1990-2016: results from the Global Burden of Diseases, Injuries, and Risk Factors 2016 Study. The Lancet Infectious Diseases 2018; 18(12): 1329-49.

2. Allwood B, van der Zalm M, Makanda G, et al. The long shadow post-tuberculosis. The Lancet Infectious Diseases 2019; 19(11): 1170-1.

3. Romanowski K, Baumann B, Basham CA, Khan FA, Fox GJ, Johnston JC. Long-term all-cause mortality in people treated for tuberculosis: a systematic review and meta-analysis. The Lancet Infectious Diseases 2019; 19(10): 1129-37.

4. Amaral AF, Coton $S$, Kato B, et al. Tuberculosis associates with both airflow obstruction and low lung function: BOLD results. European Respiratory Journal 2015; 46(4): 1104-12.

5. Byrne AL, Marais BJ, Mitnick CD, Lecca L, Marks GB. Tuberculosis and chronic respiratory disease: a systematic review. International Journal of Infectious Diseases 2015; 32: 138-46.

6. Panjabi R, Comstock G, Golub J. Recurrent tuberculosis and its risk factors: adequately treated patients are still at high risk. The International Journal of Tuberculosis and lung disease 2007; 11(8): 828-37.

7. Salomon JA, Haagsma JA, Davis A, et al. Disability weights for the Global Burden of Disease 2013 study. The Lancet Global Health 2015; 3(11): e712-e23.

8. Floyd K, Glaziou P, Houben R, Sumner T, White R, Raviglione M. Global tuberculosis targets and milestones set for 2016-2035: definition and rationale. The international journal of tuberculosis and lung disease 2018; 22(7): 723-30.

9. Menzies NA, Gomez GB, Bozzani F, et al. Cost-effectiveness and resource implications of aggressive action on tuberculosis in China, India, and South Africa: a combined analysis of nine models. The Lancet Global Health 2016; 4(11): e816-e26.

10. Organization WH. Global tuberculosis report 2019. Global tuberculosis report 2019; 2019.

11. Nemes E, Geldenhuys H, Rozot V, et al. Prevention of M. tuberculosis infection with H4: IC31 vaccine or BCG revaccination. New England Journal of Medicine 2018; 379(2): 138-49.

12. United Nations General Assembly. United to End Tuberculosis: An Urgent Global Response to a Global Epidemic. Political declaration of the high-level meeting of the General Assembly on the fight against tuberculosis. . 73rd Session of the Unites Nations General Assembly New York: WHO 2018.

13. Zak DE, Penn-Nicholson A, Scriba TJ, et al. A blood RNA signature for tuberculosis disease risk: a prospective cohort study. The Lancet 2016; 387(10035): 2312-22.

14. Wallis R, Ginindza S, Beattie T, et al. Preliminary Results of an Experimental Medicine Trial of Adjunctive Host-Directed Therapy in Adults with Moderately or Far-Advanced Rifampin-Susceptible Pulmonary Tuberculosis. C27 TB TREATMENT: American Thoracic Society; 2019: A7388-A.

15. Houben RM, Esmail H, Emery JC, et al. Spotting the old foe-revisiting the case definition for TB. The Lancet Respiratory Medicine 2019; 7(3): 199-201. 